\title{
Síndrome de Wolf-Hirschhorn por translocación: a propósito de un caso
}

\section{Wolf-Hirschhorn syndrome due to translocation: report of a case}

\author{
Eliana Alvarenga' $₫$, Elodia Torres ${ }^{\top} \odot$ \\ ${ }^{1}$ Universidad Nacional de Asunción. Instituto de Investigaciones en Ciencias de la Salud. Departamento \\ de Genética. Laboratorio de Citogenética. San Lorenzo, Paraguay.
}

\begin{abstract}
RESUMEN
El síndrome de Wolf-Hirschhorn (WHS) es una enfermedad genética causada por la pérdida de una porción distal en el brazo corto del cromosoma 4 que se caracteriza porque los pacientes presentan un rasgo craneofacial típico. Se presenta el caso de una niña de 7 meses de vida con malformaciones diversas y alimentación nasogástrica, para la realización de las técnicas de Hibridación in situ fluorescente (FISH) y cariotipo. Por FISH se detectó la microdeleción del brazo corto del cromosoma 4, región p16.3 y en el cariotipo se observó una translocación desequilibrada entre el brazo corto del cromosoma 4 y una pieza extra de origen desconocido. A fin de determinar el origen de la translocación, se realizó el estudio cromosómico a los padres, con resultados normales. Este caso revela la necesidad de la realización del FISH en pacientes con múltiples malformaciones y sospecha de ser portadores de microdeleción cromosómica.
\end{abstract}

Palabras claves: Cromosoma, genética humana.

\begin{abstract}
Wolf-Hirschhorn syndrome (WHS) is a genetic disease caused by the loss of a distal portion in the short arm of chromosome 4 . It is characterized by the fact that patients present a typical craniofacial feature. The case of a 7month-old girl with various malformations and nasogastric feeding is presented, on whom fluorescent in situ hybridization (FISH) and karyotype techniques were performed. By FISH, the microdeletion of the short arm of chromosome 4, region p16.3, was detected and in the karyotype an unbalanced translocation was observed between the short arm of chromosome 4 and an extra piece of unknown origin. In order to determine the origin of the translocation, the chromosomal study was performed on the parents, with normal results. This case reveals the need to perform FISH in patients with multiple malformations and suspected carriers of chromosomal microdeletion.
\end{abstract}

Keywords: Chromosome, human genetics.

\section{INTRODUCCIÓN}

El Síndrome de Wolf-Hirschhorn (SWH) es una enfermedad genética polimalformativa, también conocida como Síndrome $4 \mathrm{p}-$, debido a la microdeleción en la región distal del brazo corto del cromosoma 4, específicamente por la pérdida de genes contiguos localizados en $4 \mathrm{p} 16.3^{(1)}$. Fue descrito por primera vez en 1.961 por Cooper y Hirschhorn, posteriormente, en 1965 por Wolf et al. La frecuencia

Correspondencia: Eliana Alvarenga Correo: elialvarengat@gmail.com

Conflicto de interés: Los autores declaran no poseer conflicto de intereses.

Recibido: 21/10/2020 Aceptado: 03/03/2021

DOI: https://doi.org/10.31698/ped.48022021009

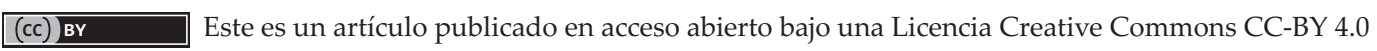


de presentación de este Síndrome es de 1 en 20.000 a 50.000 nacidos vivos, presentando el doble de frecuencia en mujeres que en varones ${ }^{(2)}$.

Los pacientes con SWH se caracterizan por tener un fenotipo facial peculiar y rasgos craneofaciales típicos en la infancia que consiste en la apariencia de "casco de guerrero griego" de la nariz (puente ancho de la nariz que continúa hasta la frente, cejas arqueadas, hipertelorismo, filtrum corto y micrognatia), discapacidad intelectual, retraso del crecimiento intrauterino y posnatal, retraso del desarrollo psicomotor, epilepsia, dificultades de alimentación, defectos cardíacos congénitos, pérdida de audición, anomalías esqueléticas, malformaciones renales y del tracto urinario y anomalías oftalmológicas y dentales ${ }^{(3)}$.

En relación a la porción del cromosoma 4 que se selecciona en estos pacientes, reportes sobre esta enfermedad, sugieren que el tamaño de la región seleccionada varía entre las personas afectadas, las deleciones más grandes tienden a provocar discapacidades intelectuales y características físicas más graves, presentan una variabilidad fenotípica en función a la gravedad. Los reordenamientos que involucran al brazo corto del cromosoma 4 y que son de novo, ocurren con mayor frecuencia en la meiosis paterna, supone en gran medida a deleciones aisladas, sin embargo, se han descrito pacientes de novo portadores de translocaciones desequilibradas entre los brazos cortos de los cromosomas 4 y 8 con alta frecuencia y de origen materno(4). La deleción $4 \mathrm{p}$ es posible detectar por citogenética convencional en asociación con el fenotipo característico, pero la microdeleción cromosómica solo es factible detectar a través de la técnica de Hibridación in situ por fluorescencia (FISH), que se utiliza para la confirmación del diagnóstico, facilitando de esa manera el tratamiento y lograr una mejor esperanza de vida de los pacientes, es por eso que el FISH se ha convertido en una herramienta importante para detectar y controlar una terapia específica para este tipo de enfermedad genética ${ }^{(5)}$.

En la Argentina, alrededor del $80 \%$ de los casos reportados son esporádicos y el $20 \%$ se originan a partir de un rearreglo familiar balanceado (Medina, Rojas, Guevara, Cañizales, \& Jaimes, 2008). Este trabajo tiene como propósito describir el caso de una niña de 7 meses de vida con sospecha clínica de ser portadora delSíndrome de Wolf-Hirschhorn.

\section{DESCRIPCIÓN DEL CASO CLÍNICO}

Se presenta el caso de una niña de 7 meses de vida derivada al Laboratorio de Citogenética del Instituto de Investigaciones en Ciencias de la Salud de la Universidad Nacional de Asunción en Paraguay (IICS - UNA), por presentar malformaciones diversas y alimentación nasogástrica, con sospecha de Síndrome de Wolf-Hirschhorn. La madre con 20 años y el padre con 26 años, sanos, no consanguíneos, refieren no haber tenido exposición a teratógenos. La niña es producto de un primer embarazo, nacida a término con 41 semanas de gestación, $2.320 \mathrm{~g}$ de peso e hipotonía generalizada. $\mathrm{Al}$ examen físico presentó microcefalia y foramen oval permeable, puente nasal chato, hipertelorismo, orejas dismórficas y de implantación baja, paladar hendido y retardo psicomotor (Figura 1 y 2). La presión pulmonar y el ritmo cardíaco normal con buena contractilidad. Para el diagnóstico citogenético, a la niña se le realizó el estudio cromosómico estándar (cariotipo) y la técnica de hibridación in situ fluorescente (FISH) para microdeleción del cromosoma 4p16. Para el FISH, se utilizó el protocolo de Sondas "Vysis" (Abbott Molecular Inc., USA), la sonda utilizada fue Vysis LSI IGH/FGFR3 DCDF para la región 4p16 spectrum Orange y 14q32 LSI IGH spectrum Green. Con las técnicas citogenéticas convencionales, se observó en el 100\% de las células analizadas una translocación desequilibrada en el brazo corto del cromosoma 4, región 4p16.3, con una pieza extra de otro cromosoma de origen desconocido, resultado el Cariotipo: 46,XX,der(4)t(4p;?)(p16.3;?) (Figura 2 y 3). Y con el FISH, en el $100 \%$ de las células analizadas se observó una señal de espectro naranja para LSI FGFR3 (4p16.3) y dos señales de espectro verde para LSI IGH (14q32), la nomenclatura resultó: ishdel(4)(p16.3)(FGFR3-) (Figura 4). Ninguna anomalía fue observada en los cromosomas de los progenitores. Cariotipo de la madre: 46,XX Cariotipo del padre: $46, X Y$. 


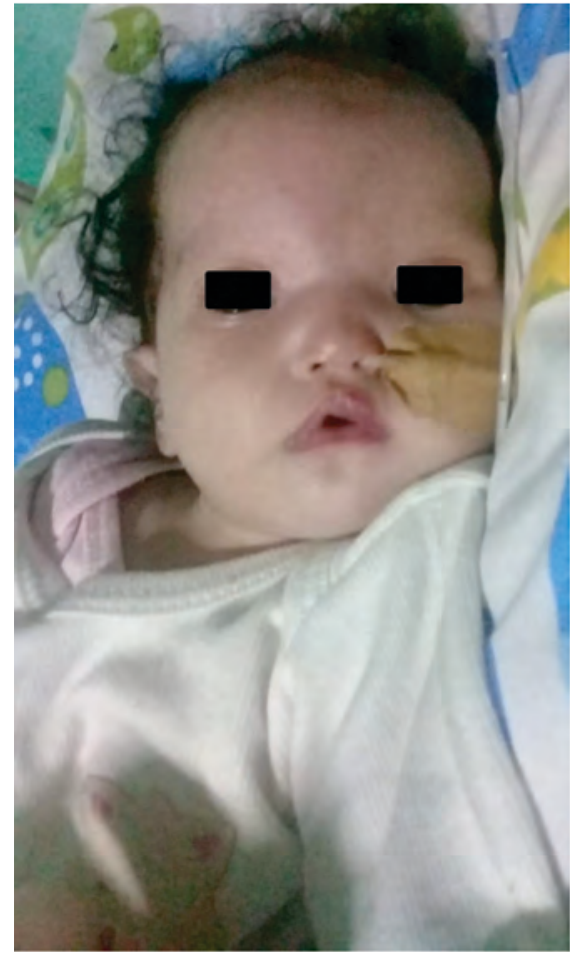

Figura 1. Se muestra la típica facies en casco de guerrero griego, caracterizado por puente nasal amplio que se extiende a la región frontal, se evidencia hipertelorismo.

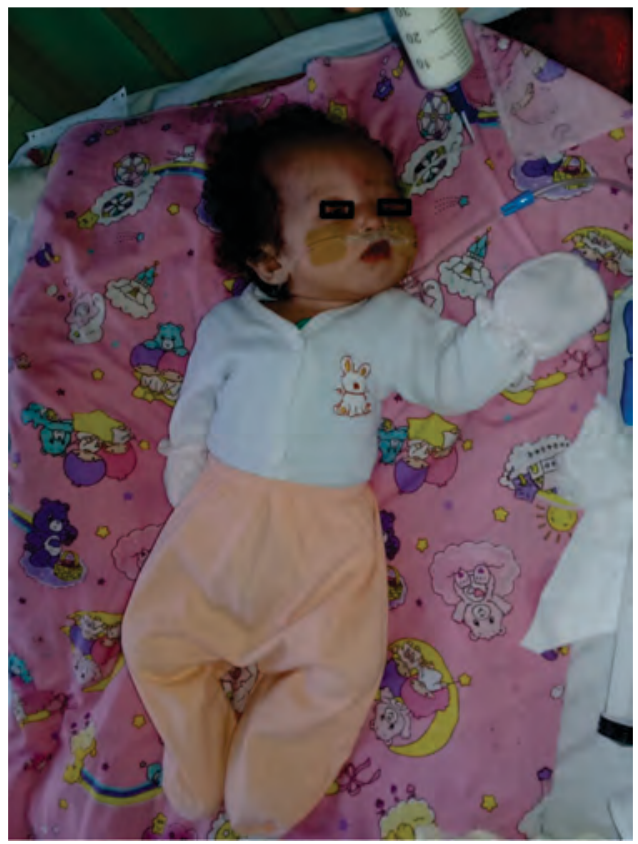

Figura 2. Microcefalia, micrognatia y orejas dismórficas.

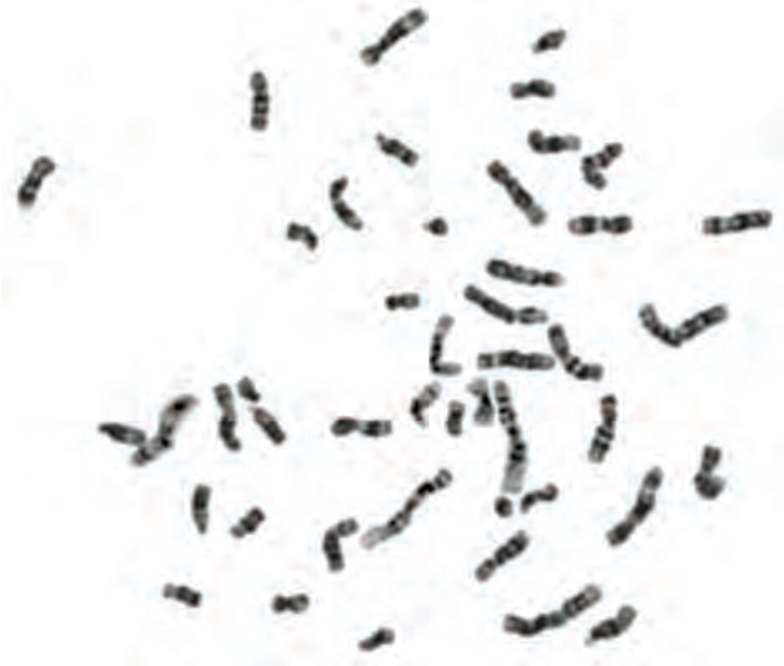

Figura 3. Metafase con Bandas G.

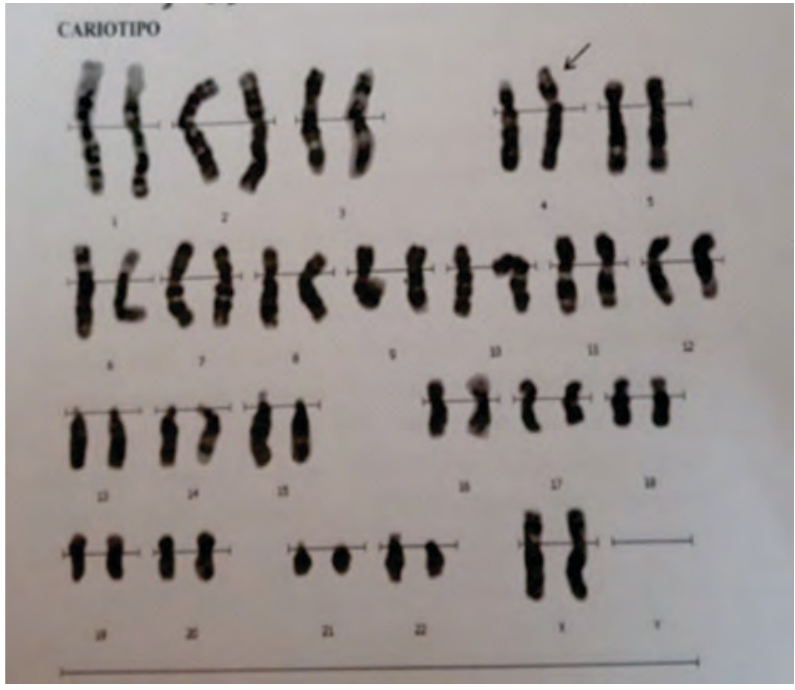

Figura 4. Kariograma donde se señala la translocación desequilibrada del brazo corto del cromosoma 4, región 4p16.3, con una pieza extra de otro cromosoma de origen desconocido. Cariotipo: 46, XX, $\operatorname{der}(4) t(4 p ; ?)(p 16.3 ; ?)$. 


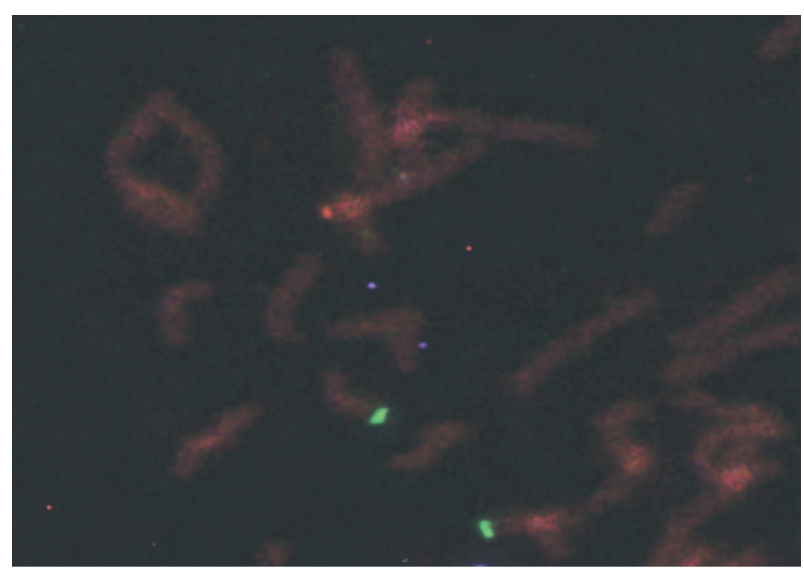

Figura 5. Se observa una señal de espectro naranja para LSI FGFR3 (4p16.3) y dos señales de espectro verde para LSI IGH (14q32). ISCN (Nomenclatura): ish del(4)(p16.3)(FGFR3-).

\section{DISCUSIÓN}

El caso que se presenta como Síndrome de Wolf Hirschhorn, enfermedad que implica deleciones de tamaño variable en la región 4p16.3, está ubicada dentro del grupo de enfermedades $\operatorname{raras}^{(6)}$. En el cariotipo de la paciente se observó que el brazo corto de uno de los cromosomas 4 se encontraba aumentado de tamaño, suponiendo la presencia de una duplicación o translocación, en el análisis de las metafases con Bandas $\mathrm{G}$, en primer lugar se descartó la duplicación porque con el FISH se detectó la microdeleción, quedando el cromosoma 4 alterado como portador de una translocación, pero no se ha podido identificar con que otro cromosoma se produjo la translocación. A fin de determinar el origen de la translocación, se procedió a realizar el cariotipo a los padres, en quienes sus cariotipos resultaron normales, por lo cual queda también establecido que se trata de un caso de novo, siendo una de las causas más frecuentes de los casos del síndrome de Wolf- Hirschhnorn ${ }^{(7)}$. Queda confirmada de esta manera la importancia de la realización de la técnica de Hibridación in situ fluorescente (FISH) en el caso de enfermedades raras, para una mayor confirmación, en el caso de una microdeleción ${ }^{(8)}$. Para estudios futuros la prueba de FISH de Painting o de Pintado cromosómico podría ser útil para determinar el origen de la translocación.

\section{AGRADECIMIENTOS}

Este trabajo corresponde a un caso analizado durante la ejecución del Proyecto de Investigación 14-INV-456 financiado por el Consejo Nacional de Ciencia y Tecnología (CONACYT -Paraguay), proyecto denominado "Frecuencia de microdeleciones cromosómicas detectadas por la técnica citogenética molecular de hibridación in situ fluorescente (FISH) en pacientes con cariotipo estándar normal". Agradecemos a la Lic. Sandra Paredes, a la MSc. Graciela Meza y al Lic. Joel Ortíz por su colaboración en el proyecto.

\section{Contribución de autoría}

\section{Eliana Alvarenga}

Redacción del manuscrito, recolección de los datos, revisión y aprobación de la versión final, asumir la corresponsabilidad de todos los aspectos del manuscrito.

\section{Elodia Torres}

Redacción del manuscrito, recolección de los datos, revisión y aprobación de la versión final, asumir la corresponsabilidad de todos los aspectos del manuscrito. 


\section{REFERENCIAS}

1. Aviña JA, Hernández DA. Síndrome de WolfHirschhorn: Microdeleción distal del brazo corto del cromosoma 4. Rev Chil pediatría [Internet]. 2008 [Citado 10 de jun 2019];79(1):50-3. Disponible en: http://www.scielo.cl/scielo.php?script=sci_arttext\&pid=S $037041062008000100007 \& \operatorname{lng}=\mathrm{en} \& \mathrm{nrm}=\mathrm{iso} \& \operatorname{lng}=\mathrm{en}$

2. Battaglia A, Carey JC, South ST. Wolf-Hirschhorn syndrome: A review and update. Am J Med Genet Part C Semin Med Genet. 2015;169(3):216-23. doi: http://doi.wiley.com/10.1002/ajmg.c.31449

3. Pokale YS, Jadhav AM, Kate U. Wolf-Hirschhorn syndrome: A case demonstrated by a cytogenetic study. Indian J Hum Genet. 2012;18(1):117-8. doi: https://doi.org/10.4103/0971-6866.96677

4. Ratan ZA, Zaman SB, Mehta V, Haidere MF, Runa NJ, Akter N. Application of Fluorescence In Situ Hybridization (FISH) Technique for the Detection of Genetic Aberration in Medical Science. Cureus. 2017;9(6):e1325. doi: https://doi.org/10.7759/cureus.1325

5. Zollino M, Lecce R, Selicorni A, Murdolo M, Mancuso I, Marangi G, et al. A double cryptic chromosome imbalance is an important factor to explain phenotypic variability in Wolf-Hirschhorn syndrome. Eur J Hum Genet. 2004;12(10):797-804. doi: https://doi.org/10.1038/sj.e jhg.5201203
6. Ho KS, South ST, Lortz A, Hensel CH, Sdano MR, Vanzo $\mathrm{RJ}$, et al. Chromosomal microarray testing identifies a $4 \mathrm{p}$ terminal region associated with seizures in WolfHirschhorn syndrome. J Med Genet. 2016;53(4):256-63. doi: https://jmg.bmj.com/lookup/doi/10.1136/jmedgenet2015-103626

7. Battaglia A. 4p (Wolf-Hirschhorn) syndrome. In: Shorvon SD, Andermann F, Guerrini R, editors. The Causes of Epilepsy [Internet]. Cambridge: Cambridge University Press; 2011[Citado 19 de jun 2019]. p. 277-80. Disponible en: https://www.cambridge.org/core/product/ identifier/9780511921001\%23c11447-41-1/type/book_part

8. Sheth F, Akinde OR, Datar C, Adeteye OV, Sheth J. Genotype-Phenotype Characterization of WolfHirschhorn Syndrome Confirmed by FISH: Case Reports. Case Rep Genet. 2012;2012:878796. doi: https://doi.org/ $10.1155 / 2012 / 878796$ 